\begin{tabular}{c} 
Terbit online pada laman web jurnal : \\
Thtp://ejournal.amikompurwokerto.ac.id/index.php/telematikal \\
Telemematikika \\
Accreditated SINTA “2” Kemenristek/BRIN, No. 85/M/KPT/2020 \\
\hline
\end{tabular}

\title{
Comparison of Inverse Kinematics and Forward Kinematics Methods on Walk Cycle Animation Characters
}

\author{
Afifah Nur Aini ${ }^{1}$, Ema Utami ${ }^{2}$, Suwanto Raharjo ${ }^{3}$ \\ 1,2 Magister Teknik Informatika Universitas Amikom Yogyakarta \\ ${ }^{3}$ Teknik Informatika, Institusi Sains \& Teknologi AKPRIND Yogyakarta \\ Email: afifah.aini@students.amikom.ac.id ${ }^{1}$, emma@nrar.net ${ }^{2}$,wa2n@nrar.net ${ }^{3}$
}

\section{A R T I C L E I N F O}

History of the article:

Received August 16, 2020

Revised September 7, 2020

Accepted February 17, 2021

\section{Keywords:}

Forward Kinematics,

Effectiveness,

$3 \mathrm{D}$,

Animation

Correspondece:

Telepon: +62 (85) 743308810

Email:

afifah.aini@students.amikom.ac.id

\begin{abstract}
The 3D animation industry is currently growing rapidly. Still, animating 3D characters is not always fast because it is often constrained at the animation stage due to the complexity or irregularity of each rig's function on the 3D character object. Therefore it takes a proper rig creation stage to support the animation process that is more efficient in-process and time. Kinematics in animation is used for reference when an object is moving. The animation uses a Kinematics approach to display natural results. This research aims to study the level of effectiveness in terms of time required to drive the 3D Walk cycle animation using the attached Kinematics \& Advanced Kinematics methods. The animation reference used was a standard human Walk cycle with the extent for each part of the body to be animated, such as head animation, hand animation, foot animation, and bed animation to complete a walking compilation of the animated Walk cycle. Each part's execution is carried out by the inverse kinematics method and then proceed with the advanced kinematics method. Based on the implementation results in each section of the walk cycle by comparing the two methods, Inverse Kinematics is an effective method for animating the legs and the head. While the Forward Kinematics method is more effective in animating the hands, body parts, and finishing movement. The results of the comparison show that the level of time effectiveness in human character $3 \mathrm{D}$ animation movements using the inverse kinematics method compared to forward Kinematics are $31.18 \%$ in body animation, $40.46 \%$ in foot animation, $13.94 \%$ in hand animation, $2.04 \%$ in head animation motion, and $7.61 \%$ for finishing walk cycle movement.
\end{abstract}

\section{INTRODUCTION}

The animation is included in one of the subsectors of a creative industry which provides for an ongoing business. Business opportunities are very promising and able to absorb large amounts of creative energy. The animation industry targets not only children but also teenagers and adults. The animation industry market can spread to various fields, including architecture and interior design, archeology, film series, advertising, education, gaming, art, and multimedia industries such as web design (Heriwati et al., 2019). Animation in the form of films during its development can be divided into several types. However, the most commonly used types are 2D animation and 3D animation (Famukhit 2016). In 2D animation, objects are drawn in flat form because they only have 2 coordinates/dimensions: the horizontal axis (X) and the vertical axis $(\mathrm{Y})$. In comparison, 3D animation has 3 coordinates, namely $\mathrm{X}, \mathrm{Y}$, and $\mathrm{Z}$, making 3D objects have volume/depth that can be seen from all directions. 
The advantage of 3D animation is that the animation creation process takes less time because the characters used are already rigged (bones) before the animation step begins. Then, at that step, the characters can be moved as desired more easily and quickly. It is different from 2D animation, which takes a long time because it is made frame by frame. Moreover, the previously created images cannot be used multiple times. Human motion is usually represented as a series of different configurations of the rigid multibody mechanism consists of a set of segments connected by joints. Segments correspond to body parts such as thighs, calves, upper arm, forearm, etc., and joints conform to articulations such as hips, knees, shoulders, elbows, etc. (Unzueta et al. 2008)

However, the process of animating 3D characters is not always fast because it is often constrained at the animation step due to the complexity or irregularity of the functions of each rig on the 3D character object. Therefore, it takes a proper rig creation step to support a more efficient animation process regarding the process and timing. Also, it requires animators to use the right method in optimizing the available time so that the animation can be completed on time, on quality, and cost in the production and post-production of $3 \mathrm{D}$ animation both in terms of the human resources that make it and the shrinkage of the computers it uses. These models are used to study the motion of objects. The rigid object model's key points are to examine the angular velocity of the rigid object, rotational motion, linear velocity, and so on. The research on particle models focuses on the equations of velocity, acceleration, deceleration, and motion of particles in a specified reference system. (Yu et al., 2017).

Through this research, the writer will focus on making 3D animation, namely rigging, which uses rig / bone animating techniques in 3D animation, known as Kinematics. Rigging is a digital framework bound to a 3D mesh. Rigging is a digital frame tied to a 3D mesh. This process uses an armature, a real skeleton that can consist of many bones (Amani and Yuly, 2019). Kinematics (Kinematics) is the study of an object's movement without considering the force that causes movement. There are two types of Kinematics in animation, and they are Forward Kinematics and inverse kinematics. Forward Kinematics can only control each segment's position indirectly by determining the angle of rotation of the joint between the base and effector tip, so the drawback of this method is that the animator must adjust the joint angle first to form a motion pose.

Conversely, with inverse kinematics, the effector tip placement can be controlled directly by breaking the joint angle, placing it in the desired location (Ge, 2000). The kinematics method is a suitable method for animation with an articulated body or parts connected to form a framework. One of the objects that can describe this form is humans, who have body parts that are connected and are connected to joints so that if there is a movement of the joint, the other connected parts will also experience a change in position, which then results in a change in position. In animation, humans are one of the most difficult characters to animate convincingly (Roberts 2013) because every part of the body moves based on anatomy, especially by a skeletal system that is interconnected with joints so that every animated movement must be done accurately and in detail. In kinesiology / biomechanics, which is the study of human body movements, humans often perform four common joint movements. The more complex the movement is, the longer it takes to make the animation. Therefore, we need a method that makes the character movement process natural and can be done easier and quicker. In this study, the author tries to provide a solution to the problems described above by making motion modeling commonly carried out by human characters as samples into 3D animation using inverse kinematics and forward kinematics methods. The kinematic 
computational quality was measured by measuring the knee angle's reconstruction during the walking and running trials. (Bernardina et al. 2019)

In this study, the author refers to several previous studies that discuss topics related to the research that the author will do. This paper will present a 3D character version using Kinematics more effectively and naturally. Research to produce a 2D animation motion model that applies the inverse kinematics method for modeling human character movements determines the degrees of freedom in several joints (Pangesti 2019). The other research aims to implement the Quick Rig feature development on Autodesk Maya, apply the inverse Kinematics and forward kinematics methods to the applied 3D animated objects, and test the effect level and the effectivities time needed. (Syalabi 2018). A research that includes virtual character control and manipulation of the underlying animation runtime, anthropomorphic hand-holding, teleoperation tasks and motion planning and trajectory generation (Starke, Hendrich, and Zhang, 2018).

\section{RESEARCH METHODS}

This study conducted observations of humans to collect data related to the animation movement of the two methods. The data obtained is then used to measure the effectiveness, which will later be used as a reference in the walk cycle character animation. Walk cycle motion kinematics seems relatively simple at first glance. Detailed observations display repetitive structures and considerable fluctuations resulting in similar but not identical repetitive cycles of motion (Vieten and Weich, 2020). The walking cycle involves a mechanism of motion. Cycling runs provide the best visual representation of the overall movement character. It encapsulates all the elements in the Principles of Animation and takes care of complex problems like weight and time (Arshad et al., 2019). Then, this research will do the grouping first and observe the application of two methods of Inverse Kinematics and Forward Kinematics. The practical step was carried out one by one so that the author would analyze each application of each method.

The data analysis results from the previous data collection step then calculated the time and motion animation parameters for each movement. After that, the author validated whether the design made is appropriate or not. The validation step is carried out with experts in their fields. This is to avoid mistakes during the later implementation. In this study, the test will be carried out by 5 professional animators who will animate 3-dimensional characters with a walk cycle reference and tested 5 times. Testing 5 times which will be counted in the later analysis, is a feasible animation result because at this step, the animator supervisor will go directly to assess and correct the animation results of each one tested by the animator. After animating the 3-dimensional character in the testing phase, comparing the effectiveness of the 3dimensional animation time that has been made using the inverse kinematic method and forward Kinematics with a reference video in the form of a live shoot video. In computer animation, similar motion optimization frameworks have been applied to generate human figure movements. (Ayusawa and Yoshida, 2018). This test aimed to find out how natural the similarities of the two videos are. Tests are carried out starting from the preparation step. Here the animator has modeling and rigging, so the animator only needs to test the processing time on each method. Next, test the time of completion or polishing of the animation section explains the research materials and the equipment used as the method. On the other hand, complexity measures usually take a lot of time (and skilled animation) to be produced by keyframing(Sanna et al., 2015). To find out how the limbs' movement during the walk cycle, an analysis of the movements was carried out through the reference video, namely the side and front directions. 
Table 1. Movement Analysis

Video Reference

The reference video comes from an endless reference entitled Athletic Male Standard Walk Animation Reference Body Mechanics, where reference video is usually used as a benchmark for making animation.



Figure 1. Research Flow

The first step is to change the Kinematics into inverse. In the picture is an example of changing the Inverse Kinematics technique on the hands and feet. The tedium of doing this procedure for each character 
for every single frame makes this process of animating a 3D character time-consuming, complicated and problematic (Bhatti et al. 2018). This is intended so that the animated movements generated from the characters can look like actual human movements by changing the kinematic. In this study, the number of motion frames made was 24 frames per second (24 FPS). The motion animation testing was conducted by 5 senior animators from MSV Studio, who had experienced being an animator for at least 10 years. The first step is to make keyframe movements, and the next is to add in-between movements between the keyframes so that the movements look smooth. In the process of making this animation, motion is using Autodesk Maya 2017 software.

\section{RESULTS AND DISCUSSION}

The step to find out how the movement of the limbs during the cycle is carried out by analyzing the movement through the reference video, namely the side and front directions. The reference video comes from an endless reference account entitled Athletic Male Standard Walk Animation Reference Body Mechanics. This reference video is usually used as a benchmark in making animation. The 3D animated character used is a free and free 3D object called Man Simple Rig, published Personal Use License by an account named banana cake. There is a description of the modifying or rigging specifications being done. The next step is implementing the 3D animation rigging/bone using the inverse kinematics method in the Autodesk Maya software. The first stage is converting Kinematics into Inverse Kinematics, then converting it again into Forward Kinematics. This is intended so that the animated movements generated from the characters can look like actual human movements by changing the kinematic. In this section, animators set a lock for the IK to handle control or joint chain. The IK / FK Key Set adds a lock for all selected control attributes for the IK handle and joint. The IK / FK button set makes for smooth animation. This option is only available if "All Fillable Attributes" is active in your options box window.

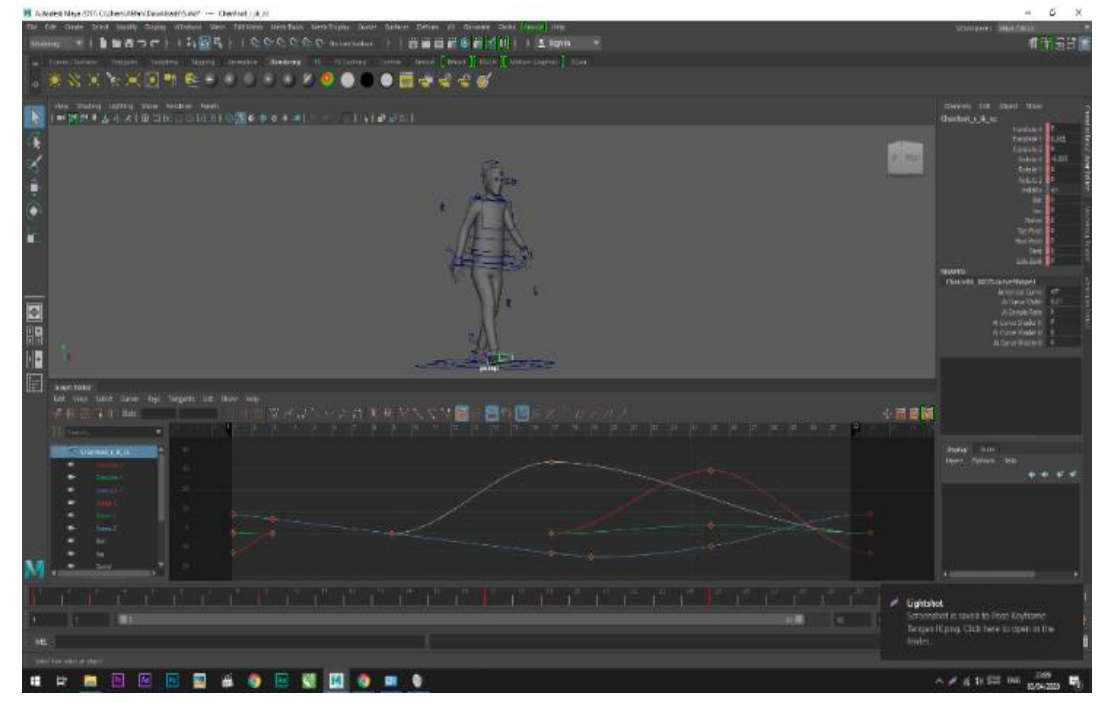

Figure 2. Kinematics on Pose Keyframe 3D IF FK

After each in-between movement is carried out, the results are presented in a table and a graph of the reference video movement test results. There is input for the FK problem is the joint angle of all joints. FK problems have unique solutions, and success depends on the connection to perform the desired transformations. The motion model's testing steps are carried out with five stages of testing for each method, both Inverse Kinematics and Forward Kinematics, namely testing on body animation, leg animation, hand animation, head animation, and finishing of animation movements using related animation principles. 
Meanwhile, input to the IK problem is a set of positions and orientations assigned to the final effector, called the target. Each end effector is given a different target, but under certain circumstances, they can coincide (Aristidou et al., 2017). Table 2 below show the result of the time each body part was tested by the animator with both methods in a matter of minutes.

Table 2. Test Result of one of the Animators

\begin{tabular}{|c|c|c|c|c|c|c|c|c|c|c|c|c|c|c|c|c|}
\hline & & & Test & tin & $(N: S$ & per & visor I & alu & & & & & & & & \\
\hline No & $M$ & $\begin{array}{l}\text { Animating } \\
\text { Walk cycle }\end{array}$ & Uji 1 & $N$ & Uji 2 & $N$ & Uji 3 & $N$ & Uji 4 & $N$ & Uji 5 & $N$ & Uji 6 & $N$ & Uji 7 & $N$ \\
\hline \multirow{5}{*}{1} & \multirow{5}{*}{ FK } & Body & 01.45 & 8 & 01.55 & 8 & 01.25 & 8 & 01.38 & 6 & 01.20 & 9 & 01.23 & 8 & 01.30 & 7 \\
\hline & & Foot & 11.05 & 7 & 10.14 & 7 & 10.13 & 7 & 10.22 & 8 & 09.40 & 8 & 10.08 & 7 & 11.20 & 6 \\
\hline & & Hand & 04.39 & 7 & 03.43 & 8 & 03.21 & 7 & 04.06 & 7 & 03.17 & 7 & & & & \\
\hline & & Head & 02.18 & 7 & 02.20 & 5 & 02.44 & 6 & 01.49 & 7 & 01.36 & 8 & 02.01 & 9 & 01.03 & 8 \\
\hline & & Finishing & 08.50 & 8 & 09.15 & 7 & 08.50 & 5 & 08.31 & 7 & 08.00 & 6 & 07.48 & 7 & 08.34 & 8 \\
\hline \multirow{5}{*}{2} & \multirow{5}{*}{ IK } & Body & 01.20 & 9 & 02.00 & 8 & 01.50 & 8 & 00.53 & 7 & 00.55 & 8 & 01.01 & 6 & 00.49 & 7 \\
\hline & & Foot & 03.06 & 8 & 03.08 & 8 & 02.43 & 9 & 02.11 & 5 & 03.52 & 7 & 04.30 & 7 & & \\
\hline & & Hand & 03.00 & 6 & 03.03 & 8 & 03.21 & 8 & 03.57 & 7 & 04.03 & 8 & 01.03 & 7 & & \\
\hline & & Head & 00.59 & 8 & 01.03 & 8 & 01.07 & 7 & 01.25 & 7 & 00.47 & 9 & 00.50 & 6 & 00.54 & 9 \\
\hline & & Finishing & 08.05 & 9 & 04.08 & 9 & 05.58 & 8 & 05.20 & 6 & 06.19 & 8 & 04.22 & 8 & & \\
\hline
\end{tabular}

After measuring each test's time by the animator, the results are presented in the form of a 3D animation motion test result table using time in seconds (s), for each part of the animation and its average for 2 methods, which is Foward Kinematics and Inverse Kinematics. The testing results on Inverse Kinematics (IK) and Forward Kinematics (FK) Hand Animation show in table 3 and table 4 below.

Table 3. Testing on Inverse Kinematics (IK) Hand Animation

\begin{tabular}{lllllll}
\hline Testing on Inverse Kinematics Hand Animation in seconds & & & \\
\hline & Test 1 & Test 2 & Test 3 & Test 4 & Test 5 & Average \\
\hline Animator 1 & 252 & 224 & 233 & 214 & 255 & 235,6 \\
\hline Animator 2 & 618 & 305 & 244 & 277 & 281 & 345 \\
\hline Animator 3 & 183 & 201 & 237 & 243 & 63 & 185,4 \\
\hline Animator 4 & 271 & 205 & 145 & 131 & 129 & 176,2 \\
\hline Animator 5 & 251 & 191 & 135 & 138 & 125 & 168 \\
\hline Average Hand Animation & & & & & 22,04 \\
\hline
\end{tabular}

Based on the results table in Table 3 above, the walk cycle animation movement on the hand part animation using Inverse Kinematics from the five test results gets an average processing time of 222.04 seconds or an average processing time of 3 minutes 42 seconds.

Table 4. Testing on Forward Kinematics (FK) Hand Animation

\begin{tabular}{lllllll}
\hline \multicolumn{7}{l}{ Testing on Forward Kinematics Hand Animation in seconds } \\
& Test 1 & Test 2 & Test 3 & Test 4 & Test 5 & Average \\
\hline Animator 1 & 156 & 140 & 145 & 135 & 170 & 149,2 \\
\hline Animator 2 & 299 & 341 & 306 & 344 & 365 & 331 \\
\hline Animator 3 & 279 & 223 & 201 & 246 & 197 & 229,2 \\
\hline Animator 4 & 111 & 126 & 135 & 124 & 114 & 122 \\
\hline Animator 5 & 131 & 146 & 135 & 124 & 84 & 124 \\
\hline Average Hand Animation & & & & & & 191,08 \\
\hline
\end{tabular}

Based on the results table in Table 4 above, the walk cycle animation movement on the hand part animation using Forward Kinematics from the five test results gets an average processing time of 191.08 seconds. Based on the time testing results on the 3D walk cycle animation of the five animators, different 
results were obtained for each animation application on the body, hands, feet, head, and finishing. Thus, the comparison testing on Forward Kinematics (FK) and Inverse Kinematics (IK) is shown in table 5 below.

Table 5. Comparassion Testing on Forward Kinematics (FK) and Inverse Kinematics (IK)

\begin{tabular}{cccc}
\hline & & \multicolumn{3}{c}{ Averages IK dan FK } \\
\hline No & & Forward Kinematics & Inverse Kinematics \\
\hline 1 & Animator 1 & 149,2 & 235,6 \\
\hline 2 & Animator 2 & 331 & 345 \\
\hline 3 & Animator 3 & 229,2 & 185,4 \\
\hline 4 & Animator 4 & 122 & 176,2 \\
\hline 5 & Animator 5 & 124 & 168 \\
\hline & Averages & 191,08 & 222,04 \\
\cline { 2 - 4 }
\end{tabular}

Based on the results in table 5 above generated from hand animation work, the time used by the two techniques does not experience a significant difference.

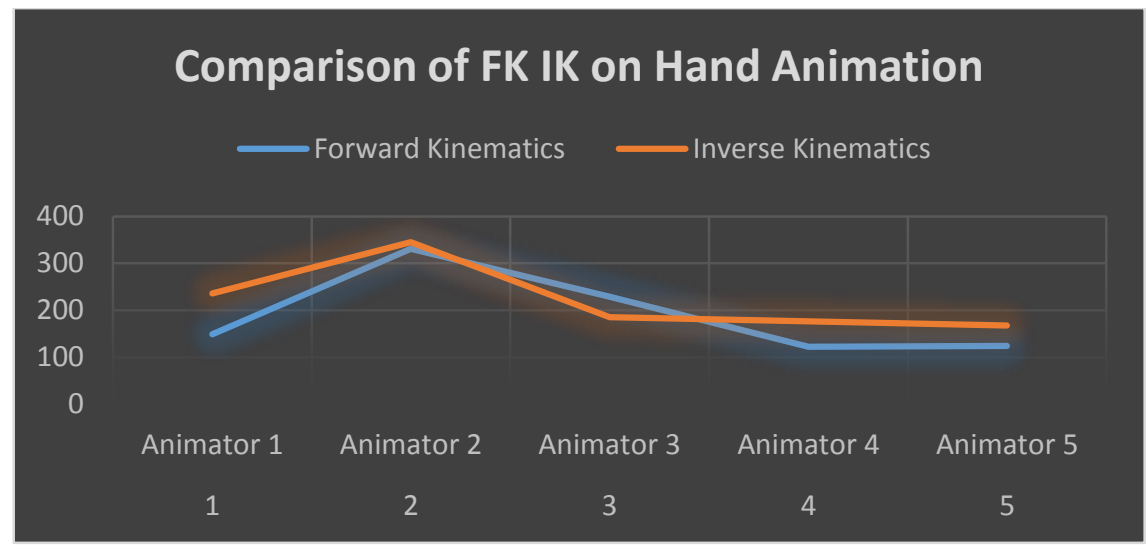

Figure 3. Graphics Comparison of FK IK on Hand Animation

Based on the results in Figure 3 above, the hand-drawn animation shows that Forward Kinematics is faster. This is shown in tables and graphs where in Forward Kinematics the average processing time is 191.08s, while in Inverse Kinematics, the time taken in body animation work is $222.04 \mathrm{~s}$. The time difference in working with the Forward Kinematics and Inverse Kinematics methods in animating the body's walk cycle is 30.96s. The difference value of each Forward Kinematics and Inverse Kinematics technique is the difference between the time difference in making the walk cycle animation for each part which is considered to be more effective. Then the calculation is done to get the percentage of the 3D animation time level, and the calculation results are described in equation 1 as follows:

$$
\begin{array}{r}
\text { Persentase }=\frac{\text { Difference in processing time }}{\Sigma \text { Total processing time }} \times 100 \\
=\frac{30.96}{222.04} \times 100=13.94 \%
\end{array}
$$

According to equation 1, to get the percentage of the 3D animation time level uses four idealized rules there are, first to find the difference of processing time (between the Forward Kinematics and Inverse Kinematics methods), second to find the total of processing time, third divided the difference of processing time by the total of processing time, fourth the result of division multiplied by 100 .

Based on the results of measuring/testing each part of the body's angle, the time taken to make the walk cycle varies depending on the controller and the movement of the body part. The angle generated by 
each joint in the 3-dimensional animation that is made has not corresponded to real human movement. The motion generated in the 3D animation produces a walk cycle motion that is slightly different from the one in the reference. This is because testing the animation motion uses the pose to pose method, making the distance between keyframes different in each motion pose. Thus, affecting the motion of the animated character 3D with motion video reference.

The inverse kinematics method is a method that can be applied to the foot part of human motion animation with an accuracy of about $40 \%$, with an average difference of two minutes of animator testing time with the forward kinematics method. By using this method, foot animation will be performed faster. However, on the other hand, it will be a little longer if you use the inverse kinematics method with an accuracy of about $13.94 \%$ with a time difference of about 30 seconds.

The supervisor in the $3 \mathrm{D}$ animation motion test also commented on the 2 techniques tested, namely testing the five parts of the body using the Inverse Kinematics technique and Forward Kinematics so many problems that caused quite a long time to work on each part of the body such as the hands using the Forward Kinematics method during the character does not interact or touch with anything else. However, if the character does some interaction, such as carrying glass or touching some items, it is better to use the Inverse Kinematics version. The rule obtained by walk cycling is that the Inverse Kinematics will be faster and more precise when used if it is locked with another object.

\section{CONCLUSIONS AND RECOMMENDATIONS}

The conclusion that can be drawn is the level of time effectiveness in human character 3D animation movements using the inverse kinematics method compared to forward Kinematics are $31.18 \%$ in body animation, $40.46 \%$ in foot animation, $13.94 \%$ in hand animation, $2.04 \%$ in head animation motion, and $7.61 \%$ for finishing walk cycle movement. So it can be concluded that the use of the inverse kinematics method can be applied to the creation of 3D animation of human movement with a faster processing time on certain body parts such as the legs and head. Human 3D animation movements created by using the Forward Kinematics method are more recommended to be applied to the hands and body.

\section{REFERENCES}

Amani, N., and A. R. Yuly. 2019. "3D Modeling and Animating of Characters in Educational Game." Journal of Physics: Conference Series 1193(1). doi:10.1088/1742-6596/1193/1/012025

Aristidou, A., J. Lasenby, Y. Chrysanthou, and A. Shamir. 2017. "Inverse Kinematics Techniques in Computer Graphics: A Survey." Computer Graphics Forum 37(6):35-58. DOI: 10.1111/cgf.13310

Arshad, Mohd Rosli, Kim Hae Yoon, Ahmad Azaini Abdul Manaf, and Muhammad Azmin Mohamed Ghazali. 2019. "Physical Rigging Procedures Based on Character Type and Design in 3D Animation." International Journal of Recent Technology and Engineering 8(3):4138-47. DOI: 10.35940/ijrte.C5484.098319

Ayusawa, Ko, and Eiichi Yoshida. 2018. "Comprehensive Theory of Differential Kinematics and Dynamics towards Extensive Motion Optimization Framework." International Journal of Robotics Research 37(13-14):1554-72. DOI: 10.1177/0278364918772893

Bernardina, Gustavo R. D., Tony Monnet, Pietro Cerveri, and Amanda P. Silvatti. 2019. "Moving System with Action Sport Cameras: 3D Kinematics of the Walking and Running in a Large Volume." PLoS ONE 14(11):1-13.

Bhatti, Zeeshan, Ahsan Abro, Abdul Rehman Gillal, and Mostafa Karbasi. 2018. "Be-Educated: Multimedia Learning through 3D Animation." ArXiv (December 2017).

Famukhit, Muga Linggar. 2016. "Simulasi Gerak Kepiting Menggunakan Metode Inverse Kinematics 
Muga Linggar Famukhit, M. Suyanto, Sukoco.” Speed, Journal Engineering, Sentra Penelitian 8(2).

Ge, Kang Teresa. 2000. "Solving Inverse Kinematics Constraint Problems for Highly Articulated Models."

Heriwati, S. H., B. H. Prilosadoso, B. Pujiono, Suwondo, and A. N. Panindias. 2019. "3D Puppets Animation for Encouraging Character Education and Culture Preservation in Surakarta." International Journal of Engineering and Advanced Technology 9(1):1551-55. DOI: 10.35940/ijeat.A1341.109119

Pangesti, Annisa Rahayu. 2019. “Analisis Penerapan Metode Inverse Kinematics Untuk Pemodelan Gerakan Karakter Manusia Pada Animasi 2 Dimensi.” Universitas AMIKOM Yogyakarta.

Roberts, Steve. 2013. Character Animation: 2D Skills for Better 3D. London: Taylor \& Francis. https://doi.org/10.4324/9780080488455

Sanna, Andrea, Fabrizio Lamberti, Gianluca Paravati, Gilles Carlevaris, and Paolo Montuschi. 2015. "Virtual Character Animations from Human Body Motion by Automatic Direct and Inverse Kinematics-Based Mapping." EAI Endorsed Transactions on Creative Technologies 2(2):e6. DOI: 10.4108/ct.2.2.e6

Starke, Sebastian, Norman Hendrich, and Jianwei Zhang. 2018. "A Forward Kinematics Data Structure for Efficient Evolutionary Inverse Kinematics." Mechanisms and Machine Science 50(April): 560-68.

Syalabi, Lalu Agam Pramadaya. 2018. “Analisis Fitur Pengembangan 'Quick Rig' Pada Autodesk Maya.” Universitas AMIKOM Yogyakarta.

Unzueta, Luis, Manuel Peinado, Ronan Boulic, and Ángel Suescun. 2008. "Full-Body Performance Animation with Sequential Inverse Kinematics." Graphical Models 70(5):87-104. https://doi.org/10.1016/j.gmod.2008.03.002

Vieten, Manfred M., and Christian Weich. 2020. "The Kinematics of Cyclic Human Movement." PLoS ONE 15(3):1-19. https://doi.org/10.1371/journal.pone.0225157

Yu, Yang, Jucheng Yang, Xiaofei Zan, Jiangang Huang, and Xiangbo Zhang. 2017. "Research of Simulation in Character Animation Based on Physics Engine." International Journal of Digital Multimedia Broadcasting 2017. https://doi.org/10.1155/2017/4815932. 